\title{
Using a Financial Calculator to Make Financial Decisions ${ }^{1}$
}

Josephine Turner ${ }^{2}$

Do you know how much your credit accounts cost you each month? Can you achieve your financial goals even if you don't work on Wall Street?

Have you ever wanted to compare savings or investment plans offering different rates of return? Have you wondered how long it would take you to save for the down payment on a house or to buy a car for cash?

Have you longed to be able to compare the cost of loans with different rates, different amounts, and different time periods? Or, have you wished that you could compare the monthly payments for different loan proposals?

Do you know how long it will take you to accumulate enough money to send the children to college? Have you wondered about the amount of money you would have available to finance your retirement if you saved $\$ 2,000$ a year? Or, would you like to know how long your savings will last if you withdraw $\$ 1,000$ per month? Have you wondered what impact inflation will have on your retirement "nest egg?"

Such problems can be solved quickly and easily using a financial calculator.* although calculations involving compound interest, periodic payments, and declining or increasing balances are complex, a financial calculator* makes them simple. Regardless of your mathematical skills and training, you can make such calculations quickly and easily. This publication shows you how.
Topics covered include:

- The Financial Calculator

- The Magic of Compounding

- Inflation

- Future Value of a Present Sum

- Present Value of a Future Sum

- Computing Retirement Payments

- Withdrawals from Investments -

o Time money will last

- Amount of money to withdraw

- How Much Money do You Need?

\section{The Math Keys}

The math function (operation) keys are located on the right of your calculator. Press the keys in order of math function desired. Think through the process and then press the appropriate keys.

Practice each math function until you feel comfortable with the pressure that must be applied to the keys to have the number appear on the display screen.

*A number of good financial calculators are on the market. This

1. This document is FCS5263/FY442, one of a series of the Department of Family, Youth and Community Sciences, Florida Cooperative Extension Service, University of Florida, UF/IFAS, Gainesville, FL 32611. First published: July 2003. Reviewed by Mary N. Harrison, professor, Department of Family, Youth and Community Sciences; and Patricia Tengel, Ph.D., CFP, Maryland. Please visit the EDIS Web site at http://edis.ifas.ufl.edu

2. Josephine Turner, Ph.D., CFP, professor, Family, Youth and Consumer Economics, Department of Family, Youth and Community Sciences, Cooperative Extension Service, UF/IFAS, Gainesville, FL 32611. 
Table 1

\begin{tabular}{|c|c|}
\hline \multicolumn{2}{|c|}{$\begin{array}{l}\text { The Financial Keys for the Hewlett Packard 10B II and/or } \\
\text { Texas Instrument BA II Plus. }\end{array}$} \\
\hline Value Keys & Command Keys \\
\hline$[\mathrm{N}][\%][\mathrm{PMT}][\mathrm{PV}][\mathrm{FV}]$ & {$\left[2^{\text {nd }}\right][\mathrm{CPT}][+/-]$} \\
\hline Key & Function of Key \\
\hline$[\mathrm{N}]$ & $\begin{array}{l}=\text { Number of periods [often } 1 \text { year } \\
\text { but could be } 1 \text { day, week or } \\
\text { month] }\end{array}$ \\
\hline $\begin{array}{l}\text { [1/y] or } \\
{[1 / \mathrm{yr}]}\end{array}$ & $=$ Interest per period \\
\hline [PV] & $=$ Present Value \\
\hline [FV] & $=$ Future value \\
\hline$[+/-]$ & $\begin{array}{l}=\text { This key is used to change the } \\
\text { sign of the number in the window }\end{array}$ \\
\hline$\left[2^{\text {nd }}\right]$ & $\begin{array}{l}\text { = On the TI BAll Plus this key } \\
\text { changes the function of keys } \\
\text { from the function written in white } \\
\text { on the key to the function written } \\
\text { in orange above the key. }\end{array}$ \\
\hline [CPT] & $\begin{array}{l}\text { = On the TI BAII Plus this key is } \\
\text { pressed to ask the calculator to } \\
\text { solve for \{compute\} whichever } \\
\text { "value" key is pressed next and } \\
\text { for end of the month payments. } \\
\text { (Press the [CPT] key and then } \\
\text { the "value" key for the unknown } \\
\text { value). }\end{array}$ \\
\hline
\end{tabular}

All five value keys may not be needed for a given problem. If you know three values, you can solve for a fourth. If you know four you can solve for the fifth. For most problems the order in which the problem is entered into the calculator will not make any difference. The only requirement is that the known values be entered before you press the [CPT] key to solve for the unknown value.

\section{The Magic of Compounding - Future Value}

The value of money changes over time. A dollar received today is worth more than a dollar received a year from now. This is the opportunity cost of postponing the use of that dollar for one year.

Compounding interest is important because it forms the basis of many financial calculations. Perhaps you are familiar with the terms "compounded annually, quarterly, or daily." These expressions mean that interest is figured on principal as frequently as the period mentioned. This interest is then added to the previous principal before the next calculation is made. Annual compounding means that this calculation is made once each year. The new interest is added to the principal before performing the next year's calculation.

Directions: Use your Financial Calculator Texas Instruments BAII Plus or Hewlett Packard 10B II to compute the following financial problems. Check answer section to see if your answers are correct.

The first example under each section provides the procedure used to solve that type of financial problem. But before we start; set your calculator. Clear memory, set decimal places, and set compounding periods to annual unless instructed otherwise. [Clear memory after each problem]

Table 2

\begin{tabular}{|c|c|c|}
\hline Set calculator & HP 10B II & TI BAll Plus \\
\hline \multirow[t]{4}{*}{ Clear memory } & \multirow{4}{*}{$\begin{array}{l}{[\mathbf{\square}][\text { clear }} \\
\text { all] }\end{array}$} & {$\left[2^{\text {nd }}\right][\mathrm{MEM}]$} \\
\hline & & {$[$ Enter $]\left[2^{\text {nd }}\right][$ Quit $]$} \\
\hline & & $\begin{array}{l}{\left[2^{\text {nd }}\right][\text { CLR work }]} \\
{\left[2^{\text {nd }}\right][\text { quit }]}\end{array}$ \\
\hline & & {$\left[2^{\text {nd }}\right][$ CLR TVM $]$} \\
\hline $\begin{array}{l}\text { Set decimal } \\
\text { place } \\
4 \text { is used in } \\
\text { this example }\end{array}$ & {$[\mathbf{\square}][\mathrm{DISP}] 4$} & $\begin{array}{l}{\left[2^{\text {nd }}\right][\text { Format }] 4[\text { Enter }]} \\
{\left[2^{\text {nd }}\right][\text { quit }]}\end{array}$ \\
\hline $\begin{array}{l}\text { Set floating } \\
\text { decimal point }\end{array}$ & {$[\mathbf{\square}][\mathrm{DISP}]$.} & $\begin{array}{l}{\left[2^{\text {nd }}\right][\text { Format }] 9[\text { Enter }]} \\
{\left[2^{\text {nd }}\right][\text { quit }]}\end{array}$ \\
\hline $\begin{array}{l}\text { Set } \\
\text { compounding } \\
\text { periods } \\
\text { Annually }\end{array}$ & $1[\boldsymbol{\nabla}][\mathrm{P} / \mathrm{YR}]$ & $\begin{array}{l}{\left[2^{\text {nd }}\right][\mathrm{P} / \mathrm{Y}] 1[\text { Enter }]} \\
{\left[2^{\text {nd }}\right][\text { quit }]}\end{array}$ \\
\hline Monthly & $12[\boldsymbol{\nabla}][\mathrm{P} / \mathrm{YR}]$ & $\begin{array}{l}\left.\left[2^{\text {nd }}\right][\mathrm{P} / \mathrm{Y}] 12 \text { [enter }\right] \\
{\left[2^{\text {nd }}\right][\text { quit }]}\end{array}$ \\
\hline
\end{tabular}

${ }^{*}[\mathrm{a}]$ is orange button

\section{Future Value of Your Savings (1-5)}

1. Over the past 100 years stock prices have appreciated on average $7 \%$ per year. If your great grandfather had left you $\$ 1,000$ in stock in 1903, what would be the value of your account today?

Table 3

\begin{tabular}{|l|l|l|}
\hline & \multicolumn{1}{|c|}{ HP 10B II } & TI BAll Plus \\
\hline Present value & $1,000[+/-][\mathrm{PV}]$ & $1000[+/-][\mathrm{PV}]$ \\
\hline Time period & $100[\mathrm{~N}]$ & $100[\mathrm{~N}]$ \\
\hline Interest rate & $7[\mathrm{I} / \mathrm{YR}]$ & $7[\mathrm{I} / \mathrm{Y}]$ \\
\hline Results & {$[\mathrm{FV}] 867,716$} & {$[\mathrm{CPT}][\mathrm{FV}] 867,716$} \\
\hline
\end{tabular}

2. Paul inherited $\$ 20,000$ from his mother. He is using this to begin his retirement fund. If he invests his inheritance at $6 \%$ annually, what will be the value in 27 years when he is ready to retire? 
3. Betty, 40 , has $\$ 15,000$ in her IRA. If the annual interest rate averages $4 \%$, how much will she have by age 62 ?

4. Betty's sister, Sarah, has $\$ 50,000$ in US Savings bonds. She plans to use this for her retirement. Sarah is currently 45 . If she receives $6 \%$ annually on the bonds, how much will she have by age 60 ?

5. Ryan has $\$ 38,000$ in a Roth IRA. He plans to retire in 12 years. How much will his IRA be worth if he receives $4 \%$ annual interest?

\section{Inflation problems (6-8)}

6. JoAnn currently lives on $\$ 1,500$ per month. This is adequate for her today. If the annual inflation rate is $4.2 \%$ how much money will JoAnn need in 15 years to maintain her current life style?

Table 4

\begin{tabular}{|l|l|l|}
\hline & HP 10B II & TI BAll Plus \\
\hline Present value & $1500[+/-][\mathrm{PV}]$ & $1500[+/-][\mathrm{PV}]$ \\
\hline Time & $15[\mathrm{~N}]$ & $15[\mathrm{~N}]$ \\
\hline Inflation rate & $4.2[\mathrm{I} / \mathrm{YR}]$ & $4.2[\mathrm{l} / \mathrm{Y}]$ \\
\hline Results & {$[\mathrm{FV}] 2,780$} & {$[\mathrm{CPT}][\mathrm{FV}] 2,780$} \\
\hline
\end{tabular}

7. James thinks that he can live on $\$ 1,200$ per month if he retired today. If the inflation rate is $5 \%$, how much will James need in 25 years to maintain today's purchasing power of $\$ 1,200$ per month?

8. A monthly retirement goal for Jim is $\$ 2,000$. If the inflation rate averages $3 \%$ per year, how much will Jim need to equal the buying power of $\$ 2,000$ in 20 years?

\section{Future Value of Regular Savings (9-11) (annual deposits)}

9. Cathy, 35 , is saving $\$ 3,000$ each year in an IRA with an interest rate of 5\% annually. What will the balance of the account be when she retires in 30 years.

Table 5

\begin{tabular}{|l|l|l|}
\hline & HP 10B II & TI BAII Plus \\
\hline Payment & $3,000[+/-][\mathrm{PMT}]$ & $3,000[+/-][\mathrm{PMT}]$ \\
\hline Time period & $30[\mathrm{~N}]$ & $30[\mathrm{~N}]$ \\
\hline Interest rate & $5[\mathrm{I} / \mathrm{YR}]$ & $5[\mathrm{I} / \mathrm{Y}]$ \\
\hline Future value & {$[\mathrm{FV}] 199,317$} & {$[\mathrm{CPT}][\mathrm{FV}] 199,317$} \\
\hline
\end{tabular}

10. Ann plans to save $\$ 1,000$ per year and put it in her IRA earning 4\% annually. Ann is now 25. If she follows her plan how much money will she have at age 65 ?

11. Fred is five years away from retirement. He has started saving $\$ 3,000$ each year. If he earns $4 \%$ annually, how much money will he have accumulated when he retires?

\section{Future Value of Savings (a lump sum and yearly additions) (12-14)}

12. George and Margean are planning to retire in 15 years. Their children are on their own and their house is paid for. Their assets include: $\$ 15,000$ in certificates of deposit, $\$ 12,000$ cash value in life insurance, and $\$ 23,000$ in IRAs. Their plan is to deposit $\$ 5,000$ annually in their retirement funds. These accounts are averaging an annual interest rate of $5 \%$. What will be the value of these assets in 15 years?

Table 6

\begin{tabular}{|l|l|l|}
\hline & HP 10B II & TI BAII Plus \\
\hline \multirow{2}{*}{$\begin{array}{l}\text { Present } \\
\text { value }\end{array}$} & & $\begin{array}{l}15,000[+] 12,000[+] \\
23,000[+]\end{array}$ \\
\cline { 2 - 3 } & $50,000[+/-][\mathrm{PV}]$ & {$[+/-][\mathrm{PV}]$} \\
\hline Payment & $5,000[+/-][\mathrm{PMT}]$ & $5,000[+/-][\mathrm{PMT}]$ \\
\hline Time period & $15[\mathrm{~N}]$ & $15[\mathrm{~N}]$ \\
\hline Interest rate & $5[\mathrm{I} / \mathrm{YR}]$ & $5[\mathrm{I} / \mathrm{Y}]$ \\
\hline Future value & {$[\mathrm{FV}] 211,839$} & {$[\mathrm{CPT}][\mathrm{FV}] 211,839$} \\
\hline
\end{tabular}

13. The balance of Nancy's tax-deferred annuity is $\$ 30,000$. If she adds $\$ 4,000$ per year for 15 years what will be the value of her account at a $4 \%$ interest rate.

14. Betsy, age 35 , has $\$ 15,000$ in her ROTH IRA. If she adds $\$ 2,000$ per year for 25 years, how much will she have if the account earns 5\% annually?

15. What would Betsy have if she deposited $\$ 3,000$ per year (new limits) instead of $\$ 2,000$ ?

Future value of savings when you make monthly deposits (16-19)

16. Ralph is 20 years from retirement. He plans to make monthly payments of $\$ 350$ to fund his retirement. If he can get an interest rate of $6 \%$, what will be the value of his account when he retires? 
Table 7

\begin{tabular}{|l|l|l|}
\hline & HP 10B II & BAll Plus \\
\hline Payment & $350[+/-][\mathrm{PMT}]$ & $350[+/-][\mathrm{PMT}]$ \\
\hline Time period & $20[\mathrm{X}] 12[=] 240[\mathrm{~N}]$ & $20[\mathrm{X}] 12[=] 240[\mathrm{~N}]$ \\
\hline Interest rate & 6[)$] 12[=] .5[\mathrm{I} / \mathrm{YR}]$ & 6[)$] 12[=] .5[\mathrm{I} / \mathrm{Y}]$ \\
\hline Future value & {$[\mathrm{FV}] 161,714$} & {$[\mathrm{CPT}][\mathrm{FV}] 161,714$} \\
\hline
\end{tabular}

17. If Bill age 42, has savings that earn an annual interest rate of $6 \%$, how much will he have by age 60 , if he saves $\$ 400$ each month?

18. Fred saves $\$ 325$ monthly; he earns $4 \%$ annually. How much will he have in 5 years?

\section{Future Value of Monthly Savings when Added to a Lump Sum.}

19. Ben has $\$ 65,000$ in his retirement fund. He will retire in 8 years. If he saves $\$ 660$ per month and adds it to his lump sum, how much will he have if he earns $5.4 \%$ annually?

Table 8

\begin{tabular}{|l|l|l|}
\hline & HP 10B II & TI BAII Plus \\
\hline Present value & $65,000[+/-][\mathrm{PV}]$ & $65,000[+/-][\mathrm{PV}]$ \\
\hline Payment & $660[+/-][\mathrm{PMT}]$ & $660[+/-][\mathrm{PMT}]$ \\
\hline Time period & $8 \mathrm{X} 12=[\mathrm{N}]$ & $8 \mathrm{X} 12=[\mathrm{N}]$ \\
\hline Interest rate & $5.4) 12=[\mathrm{I} / \mathrm{YR}]$ & $5.4) 12=[\mathrm{I} / \mathrm{Y}]$ \\
\hline Future value & {$[\mathrm{FV}] 179,055$} & {$[\mathrm{CPT}][\mathrm{FV}] 179,055$} \\
\hline
\end{tabular}

20. James and Pam have paid for their house. They plan to add the $\$ 750$ monthly they paid on the house to their $\$ 35,000$ retirement account. If they can get $6 \%$ on all these funds, how much money will they have in 5 years?

21. Joan, 40 , has saved $\$ 30,000$ in an IRA. She plans to retire at age 65 . She currently adds $\$ 200$ per month to the account. How much will she have when she retires if she averages a $6 \%$ rate of return?

How long will a retirement fund last: start with a lump sum and make monthly withdrawals (2227)

22. Delbert, age 65, has investment assets, in a deferred compensation plan, of $\$ 100,000$. If he starts taking $\$ 950$ per month from the fund to supplement his Social Security payments, how long will his fund last? Assume he has an annual interest rate of $6.25 \%$. [Reminder clear calculator.]
Table 9

\begin{tabular}{|l|l|l|}
\hline & HP 10B II & TI BAII Plus \\
\hline $\begin{array}{l}\text { Present } \\
\text { value }\end{array}$ & 100,000[PV] & 100,000[PV] \\
\hline Payments & 950[+/-][PMT] & 950[+/-][PMT] \\
\hline $\begin{array}{l}\text { Interest } \\
\text { rate }\end{array}$ & $6.25) 12=[\mathrm{I} / \mathrm{YR}]$ & $6.25) 12=[\mathrm{I} / \mathrm{Y}]$ \\
\hline $\begin{array}{l}\text { Payments } \\
\text { will last }\end{array}$ & $\begin{array}{l}{[\mathrm{N}] 152.96} \\
\text { months[) }] 12=12.75 \\
\text { years }\end{array}$ & $\begin{array}{l}{[\mathrm{CPT}][\mathrm{N}] 152.96} \\
\text { months [) }] \\
12[=] 12.75 \text { years }\end{array}$ \\
\hline
\end{tabular}

23. Patricia, 62 , has $\$ 53,000$ invested. If she makes monthly withdrawals of $\$ 800$, how long will the fund last if she averages $6.2 \%$ rate of interest?

24. Tim has a retirement fund of $\$ 103,000$. If he withdraws $\$ 1200$ per month, how long will the fund last assuming an annual interest rate of $4 \%$ ?

25. Cheryl's retirement fund has a current balance of $\$ 65,000$. She needs $\$ 550$ per month from this fund to supplement her Social Security. If she gets $5.5 \%$ how long will the fund last?

26. Sam age 70 has an IRA balance of $\$ 45,000$. If he decides to take $\$ 995$ monthly, how long will the fund last, assuming a $4 \%$ annual growth rate?

How much money can you take out each month from your nest egg? (27-30)

27. David wants to use his $\$ 96,000$ IRA to supplement his pension. How much can he take out monthly if he wants the money to last 20 years and he is getting $6 \%$ annual return?

Table 10

\begin{tabular}{|l|l|l|}
\hline & HP 10B II & TI BAIl Plus \\
\hline Present value & $96,000[+/-][\mathrm{PV}]$ & $96,000[+/-][\mathrm{PV}]$ \\
\hline Interest rate & 6[)$] 12[=][\mathrm{I} / \mathrm{YR}]$ & 6[)$] 12[=][\mathrm{I} / \mathrm{YR}]$ \\
\hline Time & $20[\mathrm{X}] 12=[\mathrm{N}]$ & $20[\mathrm{X}] 12=[\mathrm{N}]$ \\
\hline $\begin{array}{l}\text { Payment } \\
\text { amount }\end{array}$ & {$[\mathrm{PMT}] 688$} & {$[\mathrm{CPT}][\mathrm{PMT}]=688$} \\
\hline
\end{tabular}

28. Bob has $\$ 90,000$ in his retirement fund. He averages $4 \%$ annual interest rate. His life expectancy is 12.1 years. How much money can he withdraw per month if his money is to last 
29. Ruth has $\$ 150,000$ in a tax-deferred account. She wants it to last 25 years. Assume an annual interest rate of $6 \%$. (a) How much can she withdraw monthly? (b) If she decides to withdraw \$2,000 monthly, how long will the fund last? (Hint: $\$ 2,000$ is the payment)

How much money do you need to retire on? (Lump sum needed to provide a monthly income) (30-33)

30. Joe would like to withdraw $\$ 1,250$ per month for 20 years from his retirement fund. If he gets $4 \%$ annual rate of return on his money how much money will he need?

Table 11

\begin{tabular}{|l|l|l|}
\hline & HP 10B II & TI BAll Plus \\
\hline Time & $20[\mathrm{X}] 12=[\mathrm{N}]$ & $20[\mathrm{X}] 12=[\mathrm{N}]$ \\
\hline Interest & $4[\mathrm{l}] 12=[\mathrm{I} / \mathrm{YR}]$ & $4[\mathrm{)}] 12=[\mathrm{I} / \mathrm{Y}]$ \\
\hline Payment & $1250[+/-][\mathrm{PMT}]$ & $1250[+/-][\mathrm{PMT}]$ \\
\hline & {$[\mathrm{PV}] 206,277$} & {$[\mathrm{CPT}][\mathrm{PV}] 206,277$} \\
\hline
\end{tabular}

31. How much will Brenda need in her retirement fund if she plans to withdraw $\$ 600$ per month for 15 years if the account earns $5 \%$ annually?

32. How much money will Taylor need if she plans to withdraw $\$ 800$ per month for 25 years? Assume that she will get an average annual return of $4.5 \%$.

33. Jimmy would like to have his retirement fund provide a monthly income of $\$ 1,800$ for his life expectancy of 15 years. How much does he need in his retirement fund if the interest rate is only $3.6 \%$ ?

Note: the following pages contain answers to problems in this publication. 


\begin{tabular}{|c|c|c|}
\hline & \multicolumn{2}{|c|}{ Answers (rounded) } \\
\hline $2-$ & HP 10B II & TI TI BAll Plus \\
\hline Present value & $20,000[+/-][\mathrm{PV}]$ & $20,000[+/-][\mathrm{PV}]$ \\
\hline Time period & $27[\mathrm{~N}]$ & $27[\mathrm{~N}]$ \\
\hline Interest rate & $6[\mathrm{I} / \mathrm{YR}]$ & $6[\mathrm{I} / \mathrm{Y}]$ \\
\hline Results & [FV] 96,447 & [CPT][FV] 96,447 \\
\hline \multicolumn{3}{|l|}{ 3- } \\
\hline Present value & $15,000[+/-][\mathrm{PV}]$ & $15,000[+/-][\mathrm{PV}]$ \\
\hline Time period & $62[-] 40[=] 22[\mathrm{~N}]$ & $62[-] 40[=] 22[\mathrm{~N}]$ \\
\hline Interest rate & $4[\mathrm{I} / \mathrm{YR}]$ & $4[\mathrm{I} / \mathrm{Y}]$ \\
\hline Results & {$[F V] 35,549$} & [CPT][FV] 35,549 \\
\hline \multicolumn{3}{|l|}{ 4- } \\
\hline Present value & $50,000[+/-][P V]$ & $50,000[+/-][\mathrm{PV}]$ \\
\hline Time & $60[-] 45[=] 15[\mathrm{~N}]$ & $60[-] 45[=] 15[\mathrm{~N}]$ \\
\hline Interest rate & $6[\mathrm{I} / \mathrm{YR}]$ & $6[\mathrm{I} / \mathrm{Y}]$ \\
\hline Results & [FV] 119,828 & [CPT][FV] 119,828 \\
\hline \multicolumn{3}{|l|}{$5-$} \\
\hline Present value & $38,000[+/-][\mathrm{PV}]$ & $38,000[+/-][\mathrm{PV}]$ \\
\hline Time period & $12[\mathrm{~N}]$ & $12[\mathrm{~N}]$ \\
\hline Interest rate & 4 [I/YR] & $4[\mathrm{I} / \mathrm{Y}]$ \\
\hline Results & {$[F V] 60,839$} & [CPT][FV] 60,839 \\
\hline \multicolumn{3}{|l|}{$7-$} \\
\hline Present value & $1,200[+/-][P V]$ & $1,200[+/-][\mathrm{PV}]$ \\
\hline Time period & $25[\mathrm{~N}]$ & $25[\mathrm{~N}]$ \\
\hline Interest rate & 5 [I/YR] & $5[\mathrm{I} / \mathrm{Y}]$ \\
\hline Results & {$[\mathrm{FV}] 4,064$} & {$[\mathrm{CPT}][\mathrm{FV}] 4,064$} \\
\hline \multicolumn{3}{|l|}{ 8- } \\
\hline Present value & $2,000[+/-][\mathrm{PV}]$ & $2,000[+/-][\mathrm{PV}]$ \\
\hline Time period & $20[N]$ & $20[N]$ \\
\hline Interest rate & 3 [I/YR] & $3[\mathrm{I} / \mathrm{Y}]$ \\
\hline Results & [FV] 3,612 & {$[\mathrm{CPT}][\mathrm{FV}] 3,612$} \\
\hline \multicolumn{3}{|l|}{$10-$} \\
\hline Payment & $1,000[+/-][\mathrm{PMT}]$ & 1,000[+/-][PMT] \\
\hline Time period & $40[\mathrm{~N}]$ & $40[\mathrm{~N}]$ \\
\hline Interest rate & 4 [I/YR] & $4[\mathrm{I} / \mathrm{Y}]$ \\
\hline Future value & {$[F V] 95,026$} & {$[\mathrm{CPT}][\mathrm{FV}] 95,026$} \\
\hline \multicolumn{3}{|l|}{$11-$} \\
\hline Payment & $3,000[+/-][\mathrm{PMT}]$ & $3,000[+/-][\mathrm{PMT}]$ \\
\hline Time period & $5[\mathrm{~N}]$ & $5[\mathrm{~N}]$ \\
\hline Interest rate & $4[\mathrm{I} / \mathrm{YR}]$ & $4[\mathrm{I} / \mathrm{Y}]$ \\
\hline Future value & {$[F V] 16,249$} & {$[\mathrm{CPT}][\mathrm{FV}] 16,249$} \\
\hline \multicolumn{3}{|l|}{$13-$} \\
\hline Present value & $30,000[+/-][\mathrm{PV}]$ & $30,000[+/-][\mathrm{PV}]$ \\
\hline Payment & $4,000[+/-][\mathrm{PMT}]$ & $4,000[+/-][\mathrm{PMT}]$ \\
\hline Time period & $15[\mathrm{~N}]$ & $15[\mathrm{~N}]$ \\
\hline Interest rate & $4[\mathrm{I} / \mathrm{YR}]$ & $4[\mathrm{I} / \mathrm{Y}]$ \\
\hline Future value & {$[\mathrm{FV}] 134,123$} & [CPT][FV] 134,123 \\
\hline \multicolumn{3}{|l|}{$14-$} \\
\hline Present value & $15,000[+/-][\mathrm{PV}]$ & $15,000[+/-][P V]$ \\
\hline Payment & $2,000[+/-][\mathrm{PMT}]$ & $2,000[+/-][\mathrm{PMT}]$ \\
\hline Time period & $25[\mathrm{~N}]$ & $25[\mathrm{~N}]$ \\
\hline Interest rate & 5 [I/YR] & $5[\mathrm{I} / \mathrm{Y}]$ \\
\hline Future value & {$[F V] 146,250$} & {$[\mathrm{CPT}][\mathrm{FV}] 146,250$} \\
\hline
\end{tabular}

\begin{tabular}{ll} 
& \multicolumn{1}{c}{$\begin{array}{c}\text { Answers (rounded) } \\
\text { 15- }\end{array}$} \\
HP 10B II \\
Present value & $15,000[+/-][\mathrm{PV}]$ \\
Payment & $3,000[+/-][\mathrm{PMT}]$ \\
Time period & $25[\mathrm{~N}]$ \\
Interest rate & $5[\mathrm{I} / \mathrm{YR}]$ \\
Future value & {$[\mathrm{FV}] 193,977$}
\end{tabular}

TI BALL II PIus

15,000[+/-][PV]

$3,000[+/-][\mathrm{PMT}]$

$25[\mathrm{~N}]$

$5[\mathrm{I} / \mathrm{Y}]$

[CPT][FV] 193,977

$17-$

Payment $400[+/-][\mathrm{PMT}] \quad 400[+/-][\mathrm{PMT}]$

Time period $\quad 60-42=18 \times 12[N] \quad[60-42=18 \times 12=[N]$

$\begin{array}{lll}\text { Interest rate } & 6) 12=[\mathrm{I} / \mathrm{YR}] & 6) 12=[\mathrm{I} / \mathrm{Y}]\end{array}$

Future value

[FV] 154,941

[CPT][FV] 154,941

18-

Payment

Time period

Interest rate

Future value

$325[+/-][\mathrm{PMT}]$

$5 \mathrm{X} 12=[\mathrm{N}]$

4) $12=[\mathrm{I} / \mathrm{YR}]$

[FV] 21,547

$325[+/-][\mathrm{PMT}]$

$5 \mathrm{X} 12=[\mathrm{N}]$

4) $12=[\mathrm{I} / \mathrm{Y}]$

[CPT][FV] 21,547

20-

Present value

Payment

Time period

Interest rate

Future value

$35,000[+/-][\mathrm{PV}]$

750 [+/-][PMT]

$5 \times 12=[\mathrm{N}]$

6) $12=[\mathrm{I} / \mathrm{YR}]$

[FV] 99,537

$35,000[+/-][\mathrm{PV}]$
$750[+/-][\mathrm{PMT}]$

$5 \times 12=[\mathrm{N}]$

6) $12=[\mathrm{I} / \mathrm{Y}]$

[CPT][FV] 99,537

$21-$

Present value $30,000[+/-][\mathrm{PV}]$

Payment

Time period

Interest rate

Future value

200[+/-][PMT]

$65-40[=] \mathrm{X} 12=\mathrm{N}$

6) $12=[\mathrm{I} / \mathrm{YR}]$

[FV] 272,548

$30,000[+/-][\mathrm{PV}]$

200[+/-][PMT]

$65-40[=] \times 12=[\mathrm{N}]$

6) $12=[\mathrm{I} / \mathrm{Y}]$

[CPT][FV] 272,548

23-

Present value

Payment

Interest rate

Time period

53,000 [PV]

$800[+/-][P M T]$

6.2 ) $12=[\mathrm{I} / \mathrm{YR}]$

$[\mathrm{N}] 81.3) 12=[6.78$

years]

53,000[PV]

$800[+/-][P M T]$

6.2 ) $12=[\mathrm{I} / \mathrm{Y}]$

$[\mathrm{CPT}][\mathrm{N}]=81.3$

mos. $=6.78$ years

24-

Present value

Payment

Interest rate

Time period

103,000 [PV] $1200[+/-][\mathrm{PMT}]$

4 ) $12=[\mathrm{I} / \mathrm{YR}]$

[N]8.4 years

[101.28 months]

103,000[PV]

$1200[+/-][\mathrm{PMT}]$

4 ) $12=[\mathrm{I} / \mathrm{Y}]$

$[\mathrm{CPT}][\mathrm{N}]=101.28$

mos. or 8.44 years

25-

Present value

Payment

Interest rate

Time period

65,000 [PV]

$550[+/-][\mathrm{PMT}]$

5.5 ) $12=[\mathrm{I} / \mathrm{YR}]$

$[\mathrm{N}] 170.61$ months

14.22 years

65,000[PV]

$550[+/-][P M T]$

5.5 ) $12=[\mathrm{I} / \mathrm{Y}]$

$[\mathrm{CPT}][\mathrm{N}]=170.61$

mos. or 14.22

years

26-

Present value

Payment

Interest rate

Time period
45,000 [PV] 995[+/-][PMT]

4 ) $12=[\mathrm{I} / \mathrm{YR}]$

[N] 49.1 mos. $\mathrm{s}$ 4.09 years
45,000[PV]

$995[+/-][P M T]$

4 ) $12=[\mathrm{I} / \mathrm{Y}]$

$[\mathrm{CPT}][\mathrm{N}]=49.1 \mathrm{mos}$.

or 4.09 years 


\begin{tabular}{|c|c|c|c|c|c|}
\hline 28 & HP 10B II & TI BALL II Plus & 31 & HP 10B II & TI BALL II Plus \\
\hline Present value & $90,000[+/-][\mathrm{PV}]$ & $90,000[+/-][\mathrm{PV}]$ & Time & $15 \times 12=[N]$ & $15 \times 12=[\mathrm{N}]$ \\
\hline Interest rate & 4) $12=[\mathrm{I} / \mathrm{YR}]$ & 4) $12=[I / Y]$ & Interest & 5) $12=[\mathrm{l} / \mathrm{YR}]$ & 5) $12=[\mathrm{l} / \mathrm{Y}]$ \\
\hline Years & $12.1 \times 12=[\mathrm{N}]$ & $12.1 \times 12=[\mathrm{N}]$ & Payment & $600[+/-][$ PMT $]$ & $600[+/-[$ ][PMT] \\
\hline & & & & & \\
\hline $29 a$ & & & 32 & & \\
\hline \multirow[t]{2}{*}{ Present value } & $150,000[+/-]$ & $150,000[+/-][P V]$ & Time & $25 \times 12=[N]$ & $25 \times 12=[\mathrm{N}]$ \\
\hline & $\begin{array}{l}{[\mathrm{PV}]} \\
25 \times 12=[\mathrm{N}]\end{array}$ & $25 \times 12=[N]$ & Interest & $\begin{array}{l}4.5) 12= \\
4 / Y R]\end{array}$ & 4.5 ) $12=[\mathrm{I} / \mathrm{Y}]$ \\
\hline Interest & 6) $12=[\mathrm{I} / \mathrm{Y} \mathrm{Y}]$ & $\begin{array}{l}25 \times 12=[\mathrm{N}] \\
6) \quad 12=[\mathrm{I} / \mathrm{Y}]\end{array}$ & Payment & $\begin{array}{l}{[1 / \mathrm{YR}]} \\
800[/-][\mathrm{PMT}]\end{array}$ & $800[+/-][\mathrm{PMT}]$ \\
\hline \multirow[t]{2}{*}{ [PMT] } & 966.45 & $\begin{array}{l}\text { [CPT][PMT] } \\
=966.45\end{array}$ & & {$[P V] 143,928$} & [CPT][PV] 143,928 \\
\hline & & & 33 & & \\
\hline $29 b$ & & & Time & $15 \times 12=[N]$ & $15 \times 12=[\mathrm{N}]$ \\
\hline Present value & $\begin{array}{l}150,000[+/-] \\
{[\mathrm{PV}]}\end{array}$ & $150,000[+/-][\mathrm{PV}]$ & Interest & $\begin{array}{l}3.6) 12= \\
{[1 / Y R]}\end{array}$ & 3.6 ) $12=[\mathrm{I} / \mathrm{Y}]$ \\
\hline Interest rate & 6) $12=[\mathrm{I} / \mathrm{YR}]$ & 6 ) $12=[1 / Y]$ & Payment & $1800[+/-]$ & $1800[+/-][\mathrm{PMT}]$ \\
\hline Payment & 2000 [PMT] & 2000 [PMT] & & [PMT] & \\
\hline Time period & $\begin{array}{l}{[\mathrm{N}] 94.2 \text { months }} \\
\text { or } 7.85 \text { years }\end{array}$ & $\begin{array}{l}{[\mathrm{CPT}][\mathrm{N}] 94.2 \mathrm{mos} .} \\
\text { or } 7.85 \text { years }\end{array}$ & Present value & [PV] 250,068 & [CPT][PV] 250,068 \\
\hline
\end{tabular}

